\title{
Locally invasive and obstructive colonic leiomyosarcoma: a diagnostic and therapeutic challenge
}

\author{
YC Wong *, MB, BS, SY Chan, FRCS, KY Yuen, FRCS, LC Chong, FRCS \\ Department of Surgery, Tseung Kwan O Hospital, Tseung Kwan O, Hong Kong \\ * Corresponding author: kenwong18989@hotmail.com
}

Hong Kong Med J 2020;26:73-5

https://doi.org/10.12809/hkmj197873

Primary leiomyosarcoma of the gastrointestinal tract is a rare but highly aggressive neoplasm. The incidence of colonic leiomyosarcoma is approximately 0.45 cases per million population and it constitutes $0.12 \%$ of all colorectal malignancies. ${ }^{1}$ This rare malignancy possesses aggressive nature and mimicry to other relatively benign gastrointestinal diseases including gastrointestinal stromal tumour (GIST). ${ }^{1}$ Leiomyosarcoma occurs mainly in the fifth and sixth decade of life. Abdominal mass and pain are the most common presentations followed by gastrointestinal bleeding and abdominal distension. Luminal occlusion and perforation of the bowel wall are uncommon. ${ }^{2}$

Leiomyosarcoma poses a substantial preoperative diagnostic challenge to clinicians especially during emergency circumstances because there are no established diagnostic imaging criteria for leiomyosarcoma. Leiomyosarcoma presents with non-specific features on computed tomography scan. It appears non-homogeneous, irregular, and lobular-shaped with a size usually $>5 \mathrm{~cm}$, and it is associated with central necrotic areas and connection to the intestinal lumen. ${ }^{3}$ Unfortunately, histological analysis of superficial biopsy specimens has significant sampling error and leiomyosarcoma can be misdiagnosed as benign leiomyoma. The preoperative histological diagnosis of leiomyosarcoma is adequate in only $29 \%$ of rectal leiomyosarcoma cases in some studies. ${ }^{4}$ Moreover, preoperative biopsy carries a theoretical risk for tumour capsule rupture and peritoneal seeding.

The differential diagnoses for leiomyosarcoma include submucosal tumours such as adenocarcinoma, lymphoma, and GIST. Adenocarcinoma and lymphoma can be readily differentiated from leiomyosarcoma on microscopic examination, yet GISTs and leiomyosarcomas carry a lot of resemblance in both gross and histological appearance.

Immunohistochemically,

approximately 94\% of GISTs carry c-kit mutations while $95 \%$ are positive for CD117. The c-kit mutation-negative GIST is usually associated with platelet-derived growth factor receptor alpha (PDGFRA) mutations. ${ }^{5}$
In contrast, leiomyosarcoma shows features of smooth muscle differentiation with cellular atypia, abundant mitoses and areas of necrosis, reflecting their aggressive nature. Leiomyosarcoma is actin and desmin reactive but CD117, CD34 and DOG1.1 are typically absent. There is also lack of mutation of c-kit and PDGFRA genes in leiomyosarcoma. ${ }^{6-11}$

We recently encountered an exemplar case of leiomyosarcoma. A 68-year-old Chinese man presented to our hospital with progressively decreased bowel opening and abdominal distension for a few weeks. There was no abdominal pain or vomiting. He was a chronic smoker and his past health was unremarkable. His vital signs were stable and he remained afebrile during hospital stay. On physical examination, his abdomen was distended but without tenderness nor peritoneal signs. His blood test results showed leucocytosis (white blood cell count $\left.10 \times 10^{9} / \mathrm{L}\right)$. Abdominal plain X-ray showed a grossly dilated large bowel with cut-off at the descending colon, but the small bowel was not dilated. Subsequent computed tomography scan of the abdomen and pelvis with contrast revealed a $3.2-\mathrm{cm} \times 2.7-\mathrm{cm} \times 5.1-\mathrm{cm}$ obstructive mass at the lower descending colon compatible with carcinoma of the colon with an ill-defined plane between the adjacent peritoneum and the left iliacus muscle (Fig 1). Emergency Hartmann operation and en bloc resection of retroperitoneal encroaching tumour bulk involving the iliacus muscle down to the iliac crest bone were performed. Intra-operative findings included a 4-cm distal descending colonic tumour with complete obstruction and invasion to the peritoneal side wall down to the iliacus muscle and iliac crest, as well as an oedematous, grossly dilated, and faecal-loaded large bowel with competent ileocecal valve causing closed loop obstruction. Pathology report of the resected specimen revealed moderately cellular spindle cell tumour involving the muscularis propria and the extramural tissue of the colonic wall, sparing the overlying mucosa and submucosa. The tumour was composed of interlacing fascicles of spindle cells with some epithelioid appearance (Fig 2). Mitotic index was up to four mitotic figures per 10 high power fields. 

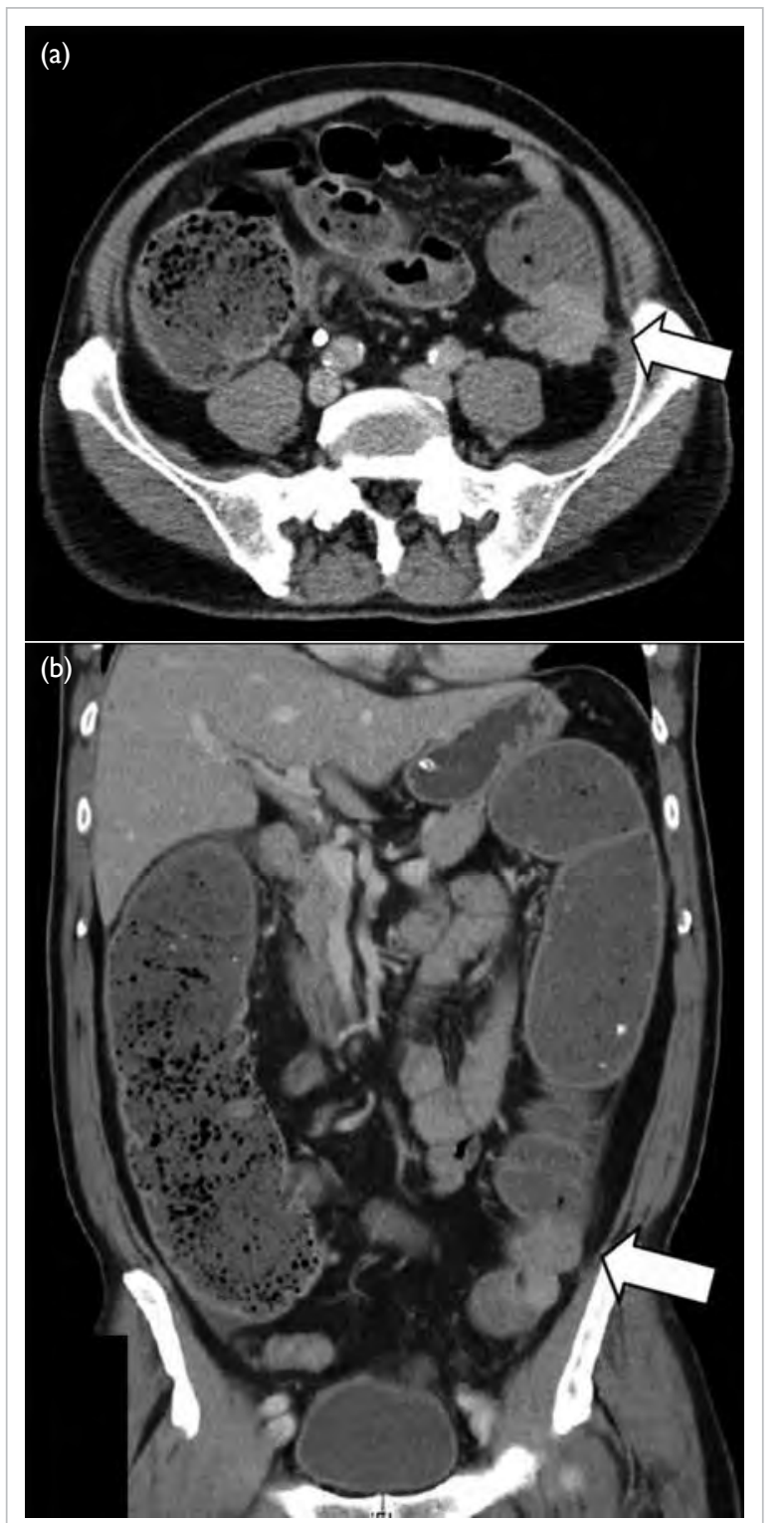

FIG I. (a) Axial and (b) coronal computed tomography images of the abdomen and pelvis, demonstrating obstructive mass at lower descending colon with invasion to the adjacent peritoneum abutting left iliacus muscle (arrows)

Immunohistochemically, the specimen was diffusely positive for desmin and actin (smooth muscle actin) and negative for MNF116, S-100, CD34, DOG-1, and c-kit mutations. The overall features were those of leiomyosarcoma. Follow-up positron emission tomography-computed tomography scan did not show any metastasis or residual tumour. Multidisciplinary consensus was reached with oncologists concluding no adjuvant chemotherapy was required. The patient had no functional deficits despite partial excision of the iliacus. The patient has attended regular follow-up examinations in our unit without recurrence.

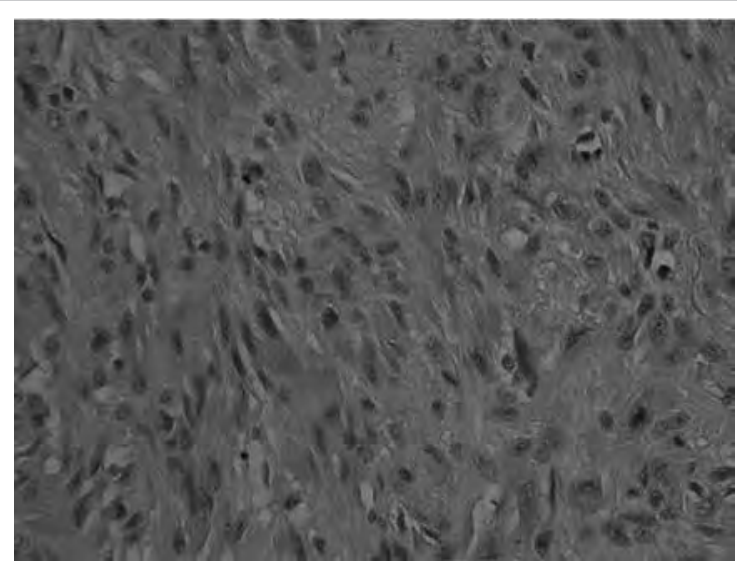

FIG 2. High-power field $(\times 400)$ shows spindle cells with oval to elongated nucleoli. The spindle cells have moderate amount of pale to amphophilic cytoplasm

Complete excision with negative margin (R0 resection) offers the best chance of cure for gastrointestinal leiomyosarcoma, and there is limited evidence supporting the use of radiotherapy and chemotherapy. En bloc excision of involved adjacent organs is necessary to achieve cure in locally advanced leiomyosarcoma. Histological grade, tumour size and location are prognostic factors, determining the likelihood of distant metastases and disease specific survival. ${ }^{12}$ In a large retrospective study, the 5-year disease-specific survival was $38.9 \%$ with a median survival of 34.1 months. Approximately $50 \%$ patients will eventually have local recurrence and distant metastases despite adequate local control of their disease. ${ }^{13}$ Continued surveillance in these patients beyond 5 years is necessary and should include imaging of the lungs along with abdomen and pelvis given hepatic and pulmonary metastases constitutes $20 \%$ and $30 \%$ of all first-site of recurrence, respectively. ${ }^{14}$

Gastrointestinal leiomyosarcoma seldom presents as intestinal obstruction and invasion to adjacent muscle as in our patient. The indistinct features of leiomyosarcoma on different imaging modalities pose substantial preoperative diagnostic challenge to clinicians. Although there is no strong evidence for the role of adjuvant therapies, curative treatment is possible with a margin-clear en bloc excision and close surveillance with imaging.

\section{Author contributions}

All authors contributed to the concept of the study, acquisition and analysis of the data, drafting of the article, and critical revision for important intellectual content. All authors had full access to the data, contributed to the study, approved the final version for publication, and take responsibility for its accuracy and integrity. 


\section{Conflicts of interest}

All authors have disclosed no conflicts of interest.

\section{Funding/support}

This commentary received no specific grant from any funding agency in the public, commercial, or not-for-profit sectors.

\section{Ethics approval}

All patients were treated in accordance with the Declaration of Helsinki. All patients provided informed consent for all procedures.

\section{References}

1. Friesen R, Moyana TN, Murray RB, Murphy F. Inglis FG. Colorectal leiomyosarcomas: a pathobiologic study with long-term follow-up. Can J Surg 1992;35:505-8.

2. Iwasa K, Taniguchi K, Noguchi M, Yamashita H, Kitagawa M. Leiomyosarcoma of the colon presenting as acute suppurative peritonitis. Surg Today 1997;27:337-44.

3. Megibow AJ, Balthazar EJ, Hulnick DH, Naidich DP, Bosniak MA. CT evaluation of gastrointestinal leiomyomas and leiomyosarcomas. AJR Am J Roentgenol 1985;144:72731.

4. Chiara O, Canini T, Segala M, Tiberio GA, Giulini SM, Tiberio G. Smooth-muscle-cell tumors of the gastroenteric tract. A review of cases [in Italian]. Minerva Chir 1997;52:1147-55.

5. Liegl B, Hornick JL, Corless CL, Fletcher CD. Monoclonal antibody DOG1.1 shows higher sensitivity than KIT in the diagnosis of gastrointestinal stromal tumors, including unusual subtypes. Am J Surg Pathol 2009;33:437-46.

6. Yamamoto $\mathrm{H}$, Handa M, Tobo T, et al. Clinicopathological features of primary leiomyosarcoma of the gastrointestinal tract following recognition of gastrointestinal stromal tumours. Histopathology 2013;63:194-207.
7. Miettinen M, Sarlomo-Rikala M, Sobin LH, Lasota J. Esophageal stromal tumors: a clinicopathologic, immunohistochemical, and molecular genetic study of 17 cases and comparison with esophageal leiomyomas and leiomyosarcomas. Am J Surg Pathol 2000;24;211-22.

8. Miettinen M, Sarlomo-Rikala M, Sorbin LH, Lasota J. Gastrointestinal stromal tumors and leiomyosarcomas in the colon: a clinicopathologic, immunohistochemical, and molecular genetic study of 44 cases. Am J Surg Pathol 2000;24:1339-52.

9. Miettinen M, Furlong M, Sarlomo-Rikala M, Burke A, Sobin LH, Lasota J. Gastrointestinal stromal tumors, intramural leiomyomas, and leiomyosarcomas in the rectum and anus: a clinicopathologic, immunohistochemical, and molecular genetic study of 144 cases. Am J Surg Pathol 2001;25:112133.

10. Miettinen M, Kopczynski J, Makhlouf HR, et al. Gastrointestinal stromal tumors, intramural leiomyomas, and leiomyosarcomas in the duodenum: a clinicopathologic, immunohistochemical, and molecular genetic study of 167 cases. Am J Surg Pathol 2003;27:625-41.

11. Miettinen M, Sorbin LH, Lasota J. True smooth muscle tumors of the small intestine: a clinicopathologic, immunohistochemical, and molecular genetic study of 25 cases. Am J Surg Pathol 2009;33:430-6.

12. Shenoy S. Primary small-bowel malignancy: update in tumor biology, markers and management strategies. J Gastrointest Cancer 2014;45:421-30.

13. Miettinen M, Fetsch JF, Sobin LH, Lasota J. Gastrointestinal stromal tumors in patients with neurofibromatosis 1: a clinicopathologic and molecular genetic study of 45 cases. Am J Surg Pathol 2006;30:90-6.

14. Clary BM, DeMatteo RP, Lewis JJ, Leung D, Brennan MF. Gastrointestinal stromal tumors and leiomyosarcoma of the abdomen and retroperitoneum: a clinical comparison. Ann Surg Oncol 2001;8:290-9. 